\title{
Design of Intelligent Recognition English Translation Model Based on Deep Learning
}

\author{
Yuexiang Ruan (i) \\ School of Foreign Studies, Shandong University of Finance and Economics, Jinan 250014, China \\ Correspondence should be addressed to Yuexiang Ruan; 20053270@sdufe.edu.cn
}

Received 14 December 2021; Revised 14 January 2022; Accepted 17 January 2022; Published 18 February 2022

Academic Editor: Naeem Jan

Copyright (@) 2022 Yuexiang Ruan. This is an open access article distributed under the Creative Commons Attribution License, which permits unrestricted use, distribution, and reproduction in any medium, provided the original work is properly cited.

\begin{abstract}
Nowadays, the intercommunication and translation of global languages has become an indispensable condition for friendly communication among human beings around the world. The advancement of computer technology developed the machine translation from academic research to industrial applications. Additionally, a new and popular branch of machine learning is deep learning which has achieved excellent results in research fields such as natural language processing. This paper improved the performance of machine translation based on deep learning network and studied the intelligent recognition of English-Chinese machine translation models. This research mainly focused on solving out-of-vocabulary (OOV) problem of machine translation on unregistered words and rare words. Moreover, it combined stemming technology and data compression algorithm Byte Pair Encoding (BPE) and proposed a different subword-based word sequence segmentation method. Using this method, the English text is segmented into word sequences composed of subword units, and, at the same time, the Chinese text is segmented into character sequences composed of Chinese characters using unigram. Secondly, the current research also prevented the decoder from experiencing incomplete translation. Furthermore, it adopted a deep-attention mechanism that can improve the decoder's ability to obtain context information. Inspired by the traditional attention calculation process, this work uses a two-layer calculation structure in the improved attention to focus on the connection between the context vectors at different moments of the decoder. Based on the neural machine translation model Google Neural Machine Translation (GNMT), this paper conducted experimental analysis on the above improved methods on three different scale datasets. Experimental results verified that the improved method can solve OOV problem and improve accuracy of model translation.
\end{abstract}

\section{Introduction}

Language is the most important bridge in communication. With rapid development for modern society and the gradual construction of global integration, the intercommunication and translation of global languages has become an indispensable condition for friendly communication among human beings all over the world. With the development of the global economy, Chinese and English have become the two most influential languages. English-Chinese translation is a shortcut to cross-language communication and plays an important role in the process of global integration. Since the development of translation business, the word-by-sent translation by human translators has long been unable to adapt to this information explosion and fast-moving society. How to transfer information more efficiently, accurately, and conveniently between various English and Chinese has become the focus of research on English-Chinese language translation. Machine translation is a kind of applied technology research in natural language processing (NLP), and it is one of the important branches. At the same time, as a benchmark subject in NLP, research on machine translation is also leading research as well as development of other branches of natural language processing [1-5].

Machine translation studies use machines to achieve automatic conversion for different languages. It highly integrates the knowledge achievements of other disciplines such as mathematics, computer science, and linguistics and is one of the most challenging subjects in NLP. In 1954, IBM cooperated with Georgetown University in the United States to use IBM-701 to complete the English-Russian translation experiment and translate 60 Russian sentences into English. 
The research of machine translation also officially began at this time. After that, the upsurge of machine translation has swept the world, and countries have launched fierce competition in machine translation. However, machine translation has suddenly encountered obstacles on the road of vigorous development. In 1966, the United States' Automatic Language Processing Advisory Committee issued a report. The report almost completely negated the prospect and value of machine translation. The report caused a serious blow to machine translation. The investment in various machine translation projects dropped sharply until it almost disappeared, and machine translation fell into a trough. Until the mid to late 1970s, with development of computer as well as the needs of economic society, machine translation ushered in recovery and prosperity. Then, after decades of development, two major categories of rule-based machine translation and statistical machine translation are formed.

Since 1990s, statistical machine translation has gradually become the mainstream. Statistical machine translation includes rule-based statistical machine translation and phrase-based statistical machine translation. Statistical machine translation enables natural language to realize automatic conversion from one language to another through the establishment of a probability model. The established probability model is trained in a large-scale parallel corpus and the model parameters are debugged. Because of this, statistical machine translation has the advantages of low labor cost, short development cycle, and better performance, which improves translation efficiency and saves translation costs. The translation platforms of well-known international companies such as Google, Baidu, and Sogou all use statistical machine translation as their core technology. But, at the same time, statistical machine translation still has many unsolvable shortcomings, such as linear inseparability, data sparseness, and inaccurate semantic representation [6-10].

The rise of deep learning has opened up a new path for the research of machine translation. Researchers have found that some techniques in deep learning can alleviate a series of problems in statistical machine translation very well. The application of deep learning technology in machine translation can be divided into two types. The first is that the main framework of the translation model is still the statistical machine translation model, but deep learning techniques will be used to improve the key modules and deficiencies in the statistical machine translation model. The second is a machine translation model based entirely on end-to-end deep neural networks proposed in 2013. This model can directly realize the automatic conversion of source language to target language. This method only relies on neural networks to handle translation problems, and a machine translation model with deep neural networks was born. At the same time, some scholars have carried out special comparison work on the effect of neural machine translation and statistical machine translation. The neural machine translation model has been verified on multiple translation tasks, and its translation performance is much higher than that of the phrase-based statistical machine translation model [11-15]. In the following paper, Section 2 presents a comprehensive discussion on the related works and reviews the literature. In Section 3, we discuss different methods and models for the machine translation processes. In Section 4, the comparative analysis is carried out through experimental discussions. In Section 5, we present the conclusion of our study.

\section{Related Work}

With the substantial increase in computer computing power, it is coupled with the expansion of data resources available on the network. As a result of the rapid development of deep learning, the field of machine translation has also undergone tremendous changes. The application of neural networks in machine translation was initially used as an auxiliary part of statistical machine translation. In terms of word alignment, literature [16] extends the hidden Markov word alignment model and uses a feedforward neural network to adjust each subcomponent. In the selection of translation rules, literature [17] uses an autoencoder to learn topic representation in the parallel corpus. By associating translation rule with information, the subject-related rules are selected based on distributed similarity with source language. In terms of sentence reordering, literature [18] uses semisupervised RAE to learn phrase representations. In terms of language models, literature [19] uses FNN to learn n-gram language models in continuous space.

With the aid of neural networks, statistical machine translation achieved the best translation results at the time. However, statistical machine translation also has shortcomings, such as data sparseness. With the increase of translation model subcomponents, training difficulties are caused. This has prompted people to explore the use of neural networks alone to achieve machine translation. Literature [20] proposed the first end-to-end encoder-decoder model structure. This model uses CNN as encoder and RNN as decoder, marking the beginning of neural machine translation. Due to the disappearance or explosion of gradients in the learning process of RNN, it is difficult to model the dependencies between the states in a long-time interval. To solve this problem, literature [21] proposed the RNN encoder-decoder model with new hidden layer nodes, and literature [22] proposed the Seq2Seq learning method, introducing LSTM into the encoder-decoder model.

Literature [23] applies the attention mechanism to the field of machine translation, and its basic idea is that the words in target language sentence are only related to some words in source language sentence. They proposed an encoder-decoder structure that combines attention, and since then machine translation has opened a new chapter. Literature [24] proposes two attention mechanisms, local and global, in NMT. Literature [25] published a translation system GNMT that combines attention mechanism and builds encoder-decoder model with LSTM. Literature [26] published a ConvS2S translation model with a translation effect comparable to RNN-based NMT but with extremely fast training speed using $\mathrm{CNN}$ structure. Although the model achieved the best translation results at the time, the limelight was quickly replaced by the Transformer model proposed in the literature [27]. The Transformer model is 
completely based on attention, and the main components are the multihead self-attention mechanism layer as well as position-by-position feedforward neural network layer. The proposal of Transformer pushes machine translation to a new level. Not only is the translation effect good, but also the model training time is shortened. Literature [28] uses the derived bilingual dictionary to initialize the translation model to achieve unsupervised machine translation. At the same time, the authors of the literature summarized the three principles of unsupervised machine translation and applied the principles to phrase-based statistical machine translation as well as neural machine translation to achieve best unsupervised machine translation results at the time.

\section{Method}

Neural machine translation model has been a hotspot in machine translation research in recent years, and its potential academic research value and commercial value are huge. With the in-depth research on NMT-related technologies, several versions of neural machine translation systems have been implemented at home and abroad. Based on the GNMT developed by Google, this chapter introduces the Seq2Seq model based on the three optimization technologies Bi-LSTM, residual network, and attention adopted by GNMT. On this basis, two improvement schemes are proposed to alleviate the OOV problem of NMT and the problem of incomplete translation.

\subsection{Neural Machine Translation Model Based on GNMT.}

The most mainstream model currently used in neural machine translation is the RNN-based Seq2Seq. The Seq2Seq model solves the common long-distance dependency problem of word sequences by introducing a special neuron LSTM. The GNMT system released by Google in 2016 also adopted this mainstream model structure and used relevant optimization techniques to optimize the model very effectively.

3.1.1. Seq2Seq Model Based on Bi-LSTM. The mainstream Seq2Seq model is based on the encoder-decoder structure of one-way RNN. In the figure, the encoder receives the embedded word vector of the source language input word sequence at the bottom layer and propagates the context of the source language input word sequence to the next hidden layer through the LSTM. The decoder initializes with the output state of the last hidden layer LSTM of the encoder and starts to predict the output of the target language according to the indication mark at the input of the decoder. Finally, the projection layer of the decoder is calculated by Beam Search, and the word sequence with the largest posterior probability is selected as the predicted translation sequence.

Generally, the embedded word vector input by the encoder carries the relevant information about the context in its forward sequence and backward sequence, but a one-way RNN can only transmit information in a certain direction. By using bidirectional RNN on the said basis, a Seq2Seq model is proposed. Figure 1 is a schematic diagram of the encoder structure constructed by the GNMT model.

In fact, the Seq2Seq model constructed by GNMT consists of an 8-layer LSTM encoder and an 8-layer LSTM decoder. The lowest layer of the encoder uses a bidirectional LSTM network. The forward network layer of the encoder processes the following information of the input word sequence from left to right, and the backward network layer processes the input word sequence from right to left and then connects the output vector sum of the two networks as a vector.

3.1.2. Seq2Seq Model Incorporating Residual Network. GNMT uses a 7-layer unidirectional LSTM and a bidirectional Bi-LSTM in an 8-layer encoder. However, simply stacking multilevel LSTM networks does not necessarily improve the translation accuracy of the model. Experience has found that when the number of stacked layers exceeds 6 , the neural network will become difficult to train and its performance will drop rapidly. This is most likely caused by gradient explosion or gradient disappearance. To remove the restriction on the number of network layers of the deep RNN, GNMT has introduced residual connections in both encoder and decoder based on the original Bi-LSTM Seq2Seq model.

Suppose that we use $L_{i}$ and $L_{i+1}$ to represent the $i$-th and $i+1$-th layers of the stacked LSTM network, respectively, and their corresponding weight parameters are represented by $a^{i}$ and $a^{i+1}$, respectively. Then the iterative calculation process of the $i$-th layer and $i+1$-th layer of the LSTM without residual connection at the current time $k$ is

$$
\begin{aligned}
c_{k}^{i}, h_{k}^{i} & =L_{i}\left(c_{k-1}^{i}, h_{k-1}^{i}, x_{k}^{i-1} ; a^{i}\right), \\
x_{k}^{i} & =h_{k}^{i} \\
c_{k}^{i+1}, h_{k}^{i+1} & =L_{i+1}\left(c_{k-1}^{i+1}, h_{k-1}^{i+1}, x_{k}^{i} ; a^{i+1}\right) .
\end{aligned}
$$

In the above equation, $c_{k}^{i}$ and $h_{k}^{i}$, respectively, represent the internal state and hidden layer state of the $i$-th layer of LSTM neurons at the current time $t$. At this time, if a residual connection is added between the output of the $i$-1-th layer and the output of the $i$-th layer, the iterative calculation process of the entire neuron state is changed to

$$
\begin{aligned}
c_{k}^{i}, h_{k}^{i} & =L_{i}\left(c_{k-1}^{i}, h_{k-1}^{i}, x_{k}^{i-1} ; a^{i}\right), \\
x_{k}^{i} & =h_{k}^{i}+x_{k}^{i-1}, \\
c_{k}^{i+1}, h_{k}^{i+1} & =L_{i+1}\left(c_{k-1}^{i+1}, h_{k-1}^{i+1}, x_{k}^{i} ; a^{i+1}\right) .
\end{aligned}
$$

It can be seen that, on the original basis, the output items of the low-level LSTM are added. The addition of the residual connection layer allows the reverse gradient of the neural network to easily propagate from the $i$-th layer to the $i$-1-th layer. This method significantly improves the flow ability of the gradient in the back-propagation. This effectively reduces the explosion and disappearance of gradients in deep neural networks, making it a reality to build deep neural network structures. 


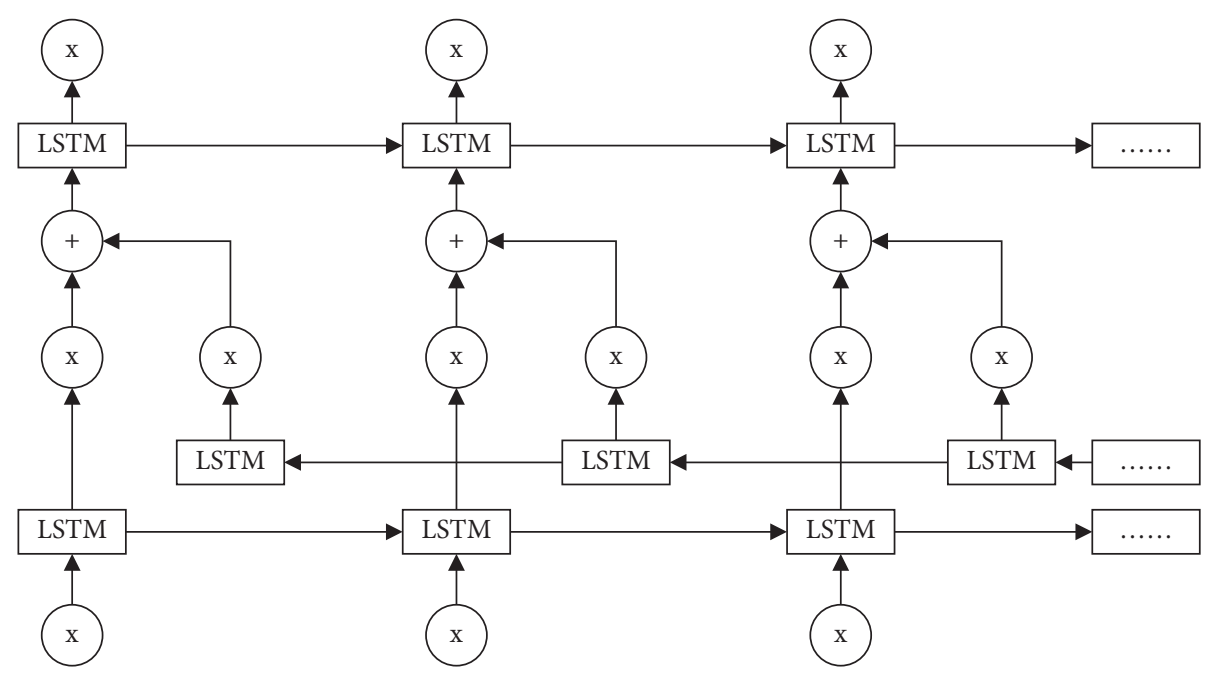

Figure 1: Seq2Seq model based on Bi-LSTM.

3.1.3. Seq2Seq Model Incorporating Attention Mechanism. Literature [36] proposed two different attention vector calculation methods, global attention and local attention. Global attention uses the output state of all hidden layers of the highest encoder layer to participate in the calculation. Local attention only selects the local state aligned with the current hidden layer of the decoder to participate in the calculation of the context vector, eliminating the defect of excessive calculation of global attention on long text.

To enable the decoder to be calculated in parallel on multiple LSTM layers as much as possible, GNMT has changed the method used by the above two to obtain the hidden state from the LSTM at the top of the decoder. Instead, it directly obtains the hidden state from the lowest layer of the decoder st 1 and adds it to the calculation of the attention vector. Figure 2 shows the complete Seq2Seq model structure of GNMT incorporating the attention mechanism. The entire attention calculation process is still the same as the original calculation process. The GNMT model uses a fully connected feedforward neural network with a single hidden layer as the calculation function of the attention vector.

\subsection{Neural Machine Translation Model Based on Improved} GNMT. Based on the GNMT model, this paper proposes two optimization schemes to improve the GNMT model from the perspective of solving the OOV (out-of-vocabulary) problem of NMT and the phenomenon of incomplete translation.

3.2.1. Improved Word Sequence Segmentation Method. English word segmentation usually uses spaces between words for segmentation, while Chinese uses characters as the basic unit. Therefore, the word segmentation method is relatively complicated. There are many Chinese word segmentation tools such as Jieba, THULAC, and HanLP that can help us achieve word segmentation. Although the word segmentation methods of the above two languages are different, their common point is that the segmentation of word sequences in the two languages is based on the word level.

The word-based sentence segmentation method is intuitive and easy to understand and conforms to the human cognitive model of language, but its drawback is that it will produce a large-scale vocabulary. Taking the dataset NLPCC2019 as an example, we segmented 4.79 million aligned Chinese and English sentence pairs. A total of 929,220 Chinese words and 815,978 English words were generated. However, due to the limitation of computing performance, the size of the vocabulary usually cannot be too large. The fixed-size vocabulary usually used is $30 \mathrm{k} \sim 50 \mathrm{k}$. If we limit the size of the Chinese and English vocabulary to $40 \mathrm{k}$, then, in the Chinese and English vocabulary of the dataset NLPCC2019, the word frequency of the English vocabulary with the lowest frequency is 65 , and the word frequency of the Chinese vocabulary is 111 . Words below this frequency will be replaced with unregistered words UNK in the dataset, which affects the accuracy of the NMT translation model to a certain extent.

To alleviate the OOV problem caused by a fixed-scale vocabulary, the researchers proposed two types of solutions. We know that named entities such as English person names and place names are usually literally translated into Chinese text. Therefore, the first type of solution is to directly copy the unregistered words from the source language to the translation in the target language. Based on this idea, literature [29] proposed a solution to copy the text of rare words using the attention model. However, because the attention mechanism of deep neural networks is not stable and reliable, this method of simply copying the source language text to solve the OOV problem has certain limitations. The second type of solution is to decompose the word sequence of the sentence pair into a string sequence composed of morpheme subword units. For example, the document [30] proposed separating words into a sequence of characters with smaller structures. Literature [31] proposed HybridNMT combining word and character. 


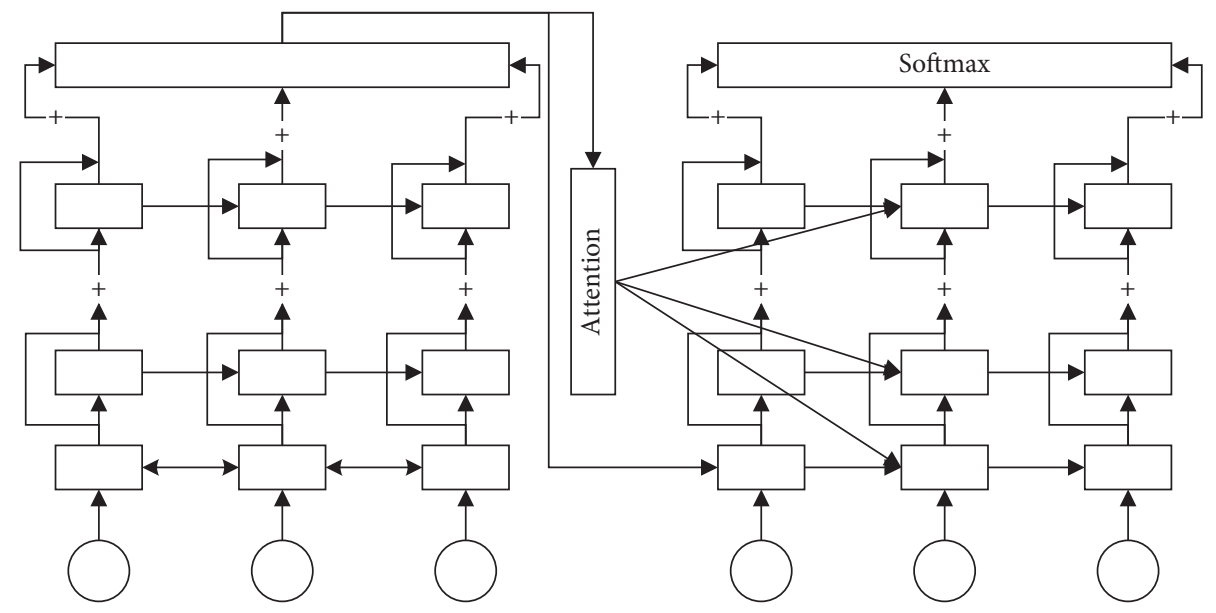

FIgURE 2: Seq2Seq model incorporating attention mechanism.

Literature [32] used the data compression algorithm BPE [33] to segment the original word into a string composed of morphemes and subwords. Although the character sequence based on character greatly reduces the time spent on data preprocessing, it also increases the length of the sequence, thus increasing the time and complexity of model training. Although HybridNMT based on a mixture of word and character can use more corpus, it cannot ensure the correctness of word separation. The subword segmentation based on the BPE compression algorithm balances the relationship between the size of the vocabulary and translation efficiency.

Based on subword, this paper proposes a word sequence segmentation method that combines stemming and BPE algorithm to solve the OOV problem that often occurs in open vocabularies.

(1) Stemming Technology. English belongs to the European language, and the same root of English words usually contains many variants, such as the singular and plural of nouns and the conjugation of verbs in different tenses. It can be seen that English contains a large number of inflections or compound words based on the same root. Stemming technology is the process of removing the affixes of inflection words or compound words to obtain the root of the word. The use of stemming technology can effectively reduce a part of the vocabulary without losing most of the semantics.

(2) Byte Pair Encoding and Stemming. Byte Pair Encoding (BPE) is a data compression algorithm. Its core idea is to replace the bytes pair with the highest frequency in the sequence with a new byte during each iteration. The one-iteration process of the BPE algorithm is described as follows. First, calculate the total frequency of adjacent cooccurring character pairs in the sequence in the vocabulary. Repeat the above statistics on all character sequences to obtain a set consisting of cooccurring character pairs and their total frequencies. Then select the character pair with the highest occurrence frequency in the set pairs, merge them, and finally update the corresponding character sequence in the vocabulary to complete an iteration process. To prevent the high- frequency words in the original vocabulary from being segmented into morpheme subwords, we can set a threshold to ensure that words with a frequency higher than the threshold are not segmented and save this part of the word in the vocabulary. Combining the stemming technology, to avoid the polysemy problem caused by the use of stemming, all words in the range of the high-frequency word shortlist will not be stemming. All other words that are not in the shortlist will be replaced with stems, and a new dataset will be generated after the replacement.

Based on the above explanation, this article adopts a different word sequence segmentation scheme from GNMT. For the processing of English word sequences, we adopted a new word sequence segmentation scheme combining stemming and BPE. For the Chinese word sequence, a word segmentation method based on the unigram model is used to directly segment the Chinese sentence into characters. This segmentation method can significantly reduce the vocabulary of the Chinese vocabulary.

3.2.2. Improved Attention Structure. Using the attention mechanism is a very effective method for NMT to process long sentence translation. The reason is that using the attention mechanism can improve the ability of the NMT decoder to obtain the context information of the encoder and assist the decoder in making output predictions. Since attention was proposed, many variants have been derived.

To solve the incomplete translation problem of GNMT and further improve the decoder's ability to obtain context information, this paper proposes a new and improved attention calculation method to assist the calculation of the GNMT speculation layer.

The NMT decoder has three input values involved in the calculation at the hidden layer output $s_{k}$ at the current time $k$. They are the hidden layer output state $s_{k-1}$ of the decoder at the previous moment, the predicted output $y_{k-1}$ of the decoder at the previous moment, and the context vector $c_{k}$ at the current moment $t$ generated by the attention calculation.

We noticed that the decoder has a corresponding context vector $c_{k}$ that carries important contextual information at each 
time $k$, and there must be some connection between the context vectors at different times. Considering that the semantic structure of the source language and the target language may be very different, the word sequence of encoder and decoder may not have the same context structure. Therefore, the context vector with the highest correlation with the current moment $c_{k}$ is not necessarily $c_{k-1}$. When focusing on the context vector at the current moment, we need to refer to all previous context vectors that are close to it at the same time to ensure that current $c_{k}$ can obtain the most accurate context information. Based on this idea, we give an improved attention structure that uses a deeper attention calculation to increase the attention to context information.

Assuming that the original context vector corresponding to the decoder from time 0 to time $k$ is $C=\left(c_{1}, c_{2}, \ldots, c_{k}\right)$, the context vector that has been improved and updated is $\widehat{c}=\left(\widehat{c_{1}}, \widehat{c_{2}}, \ldots, \widehat{c_{k}}\right)$. We define the correlation between the current context vector $c_{k}$ and the historical context vector $c_{h}$ as follows:

$$
d_{h}=G\left(c_{k}, c_{h}\right)
$$

Function $G$ is a function mapping that measures the correlation between vectors, and $d_{h}$ is called the correlation coefficient of the vector. According to mathematical experience, function $G$ can be defined as the angle cosine operation of a vector, the distance of a vector, or the cross entropy of a vector. Similar to the calculation process of the attention vector, the correlation coefficient $d_{h}$ needs to be normalized to a real number in the range of $0 \sim 1$.

$$
\alpha_{h}=\frac{\exp (d)}{\sum_{i=1}^{k-1} \exp \left(d_{i}\right)} \text {. }
$$

Vector $\alpha=\left(\alpha_{1}, \alpha_{2}, \ldots, \alpha_{h}\right)$ composed of $t$ correlation coefficients is called the correlation vector of the context vector. Let $c_{k}^{\prime}=\sum_{i=1}^{k-1} \alpha_{i} c_{i}$; then the calculation formula of the context vector $\widehat{c_{k}}$ at the current moment of improving attention is

$$
\widehat{c_{k}}=c_{k}+\sum_{i=1}^{k-1} \alpha_{i} c_{i} .
$$

It can be seen that the calculation of the improved context vector $\widehat{c_{k}}$ comprehensively considers the context vector $c_{k}$ at the current moment and the weighted and quantized historical context vector $c_{k}^{\prime}$. Therefore, the hidden state of decoder at time $t$ is calculated as

$$
s_{k}=f\left(y_{k-1}, s_{k-1}, \widehat{c_{k}}\right) \text {. }
$$

Since this article is based on GNMT's Seq2Seq model, it incorporates an improved deep attention.

\section{Experiment and Discussion}

In this section, we carried out the comparative analysis for the proposed methods and the existing ones. To this end, we performed different experiments and studied the experimental results carefully. As a result, it was verified that the proposed methods stand out as compared to the rest of the techniques.
4.1. Dataset. This paper uses two different scale datasets NLPCC2019 and OPUS to train, verify, and test the NMT model. The Chinese and English parallel corpora from three different sources are all downloaded from the Internet, and they are publicly available and free data materials. OPUS dataset is derived from the translation of Chinese and English movie subtitles, which contains 9,304778 aligned Chinese and English sentence pairs, with a total data size of $1.17 \mathrm{~GB}$. After observation, it was found that the Chinese translation part contained garbled characters. Some Chinese translations are mixed with English original sentence information, which will interfere with translation. Some Chinese translations contain obvious translation errors. After data preprocessing and screening, we eliminated this part of the data, and a total of 7,256,783 pairs of sentences in Chinese and English were obtained. NLPCC2019 dataset provides 5.2 million aligned Chinese and English sentence pairs. The text corpus comes from various industries and the total data size is $1.1 \mathrm{~GB}$. After sampling and analysis, a small number of sentence pairs in this dataset have translation errors. We screened the dataset on a large scale and selected $2,714,662$ high-quality sentence pairs. According to statistics, in the filtered text corpus, the average length of Chinese sentences is 19 words, and the average length of English sentences is 37 words. There are few long sentences in the corpus, and we limit the maximum length of sentences to less than 100. Table 1 shows a simple comparison of the two datasets.

4.2. Experimental Environment. The experimental part of this article is carried out on the Ubuntu operating system, using the currently very popular deep learning framework PyTorch, combined with GPU for experimental model building and calculation. The details of the experimental environment are shown in Table 2. The evaluation metric is BLEU.

4.3. Evaluation on Network Training. In a deep learning network, one of the most important indicators is the convergence of the network. To evaluate whether the network designed in this paper can effectively converge on the dataset, this work analyzes the training loss and BLEU score. The results are shown in Figures 3 and 4.

It can be seen from the two figures that as the training progresses, the training loss gradually decreases, and the training BLEU gradually increases. On NLPCC2019 and OPUS, when the training epoch reaches 300 , the loss value almost no longer decreases, and the BLEU almost no longer rises, which indicates that the deep learning network has reached a state of convergence. It also illustrates the reliability and effectiveness of the network designed in this project.

4.4. Comparison with Other Methods. To verify the validity and correctness of the method designed in this work, we compare the method in this paper with other English machine translation methods. The methods compared are 
TABLE 1: Comparison of detailed information of two datasets.

\begin{tabular}{lcccr}
\hline Dataset & Language & Train (M) & Test (K) & Number \\
\hline NLPCC2019 & English & 2.55 & 60 & 359482 \\
CPUS & $\begin{array}{l}\text { Chinese } \\
\text { English } \\
\text { Chinese }\end{array}$ & 7.25 & 105 & 201505 \\
& & & 4202 \\
\end{tabular}

TABle 2: Experimental environment.

\begin{tabular}{lr}
\hline Item & Version \\
\hline Operating system & Ubuntu 20.04 \\
GPU & NVIDIA GeForce GTX 1080 Ti \\
PyTorch & PyTorch 1.7 \\
Python & Python 3.7 \\
Memory & $8 \mathrm{G}$ \\
\hline
\end{tabular}
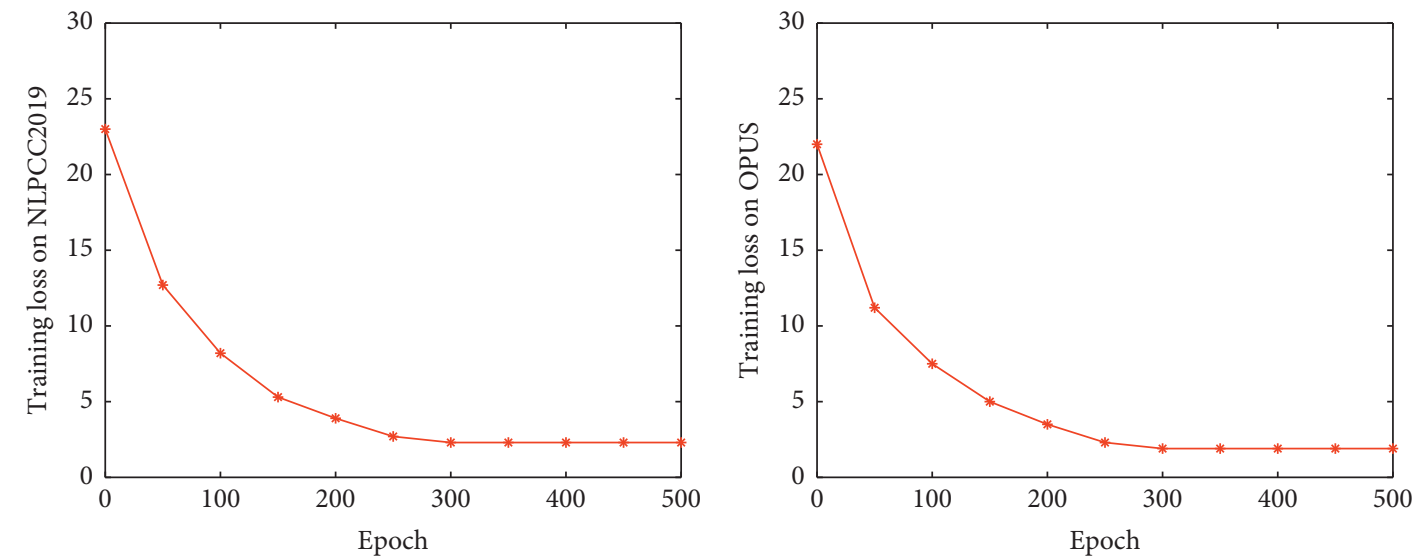

Figure 3: The training loss on two datasets.
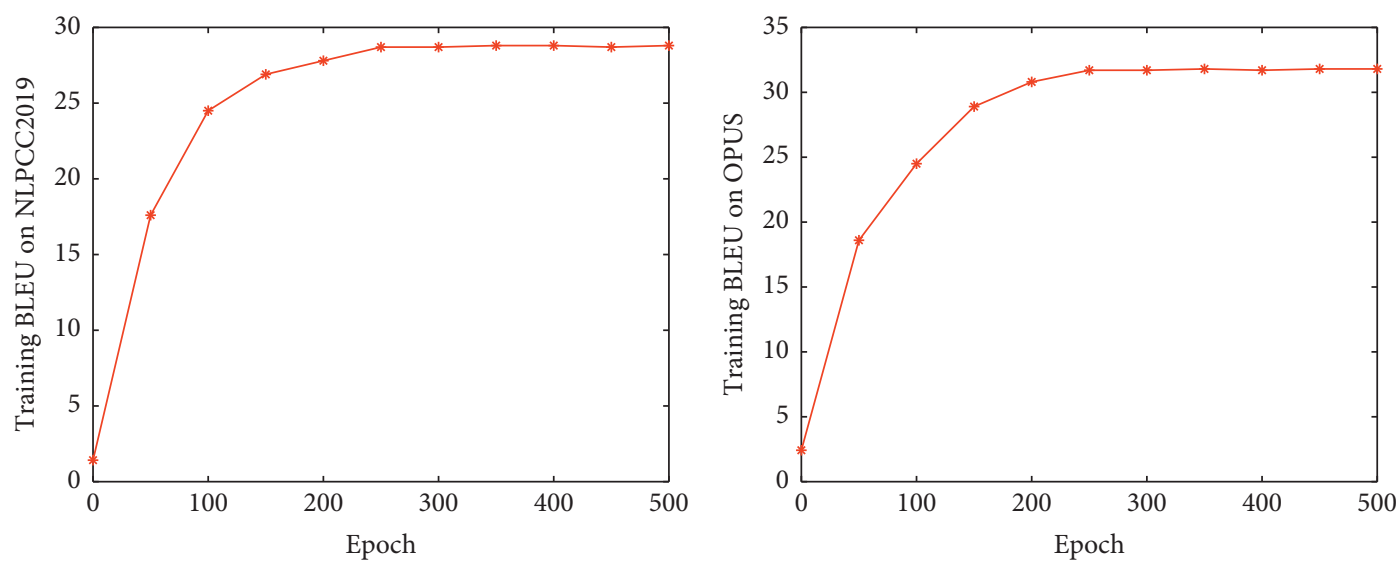

FIgURE 4: The training BLEU on two datasets.

RNN-Based, LSTM-Based, Seq2Seq-Based, and AttentionBased methods. The experimental results are shown in Table 3.

It can be seen that, compared with the other methods listed in the table, the improved GNMT (IGNMT) method designed in this article can obtain the best performance on the two datasets. In NLPCC2019 dataset, compared with the best performing method, IGNMT can achieve a $1.1 \%$ increase in BLEU. On OPUS, compared with the best performing method, IGNMT can achieve a $1.5 \%$ increase in BLEU. It can be proved that the method proposed in this paper can achieve advanced performance, and it also proves the effectiveness of the method proposed in this work. 
TABLE 3: Comparison with other methods.

\begin{tabular}{lcc}
\hline Method & NLPCC2019 & OPUS \\
\hline RNN-Based & 12.7 & 13.4 \\
LSTM-Based & 14.4 & 14.9 \\
Seq2Seq-Based & 16.2 & 16.8 \\
Attention-Based & 17.4 & 18.2 \\
IGNMT (Ours) & 18.5 & 19.7 \\
\hline
\end{tabular}

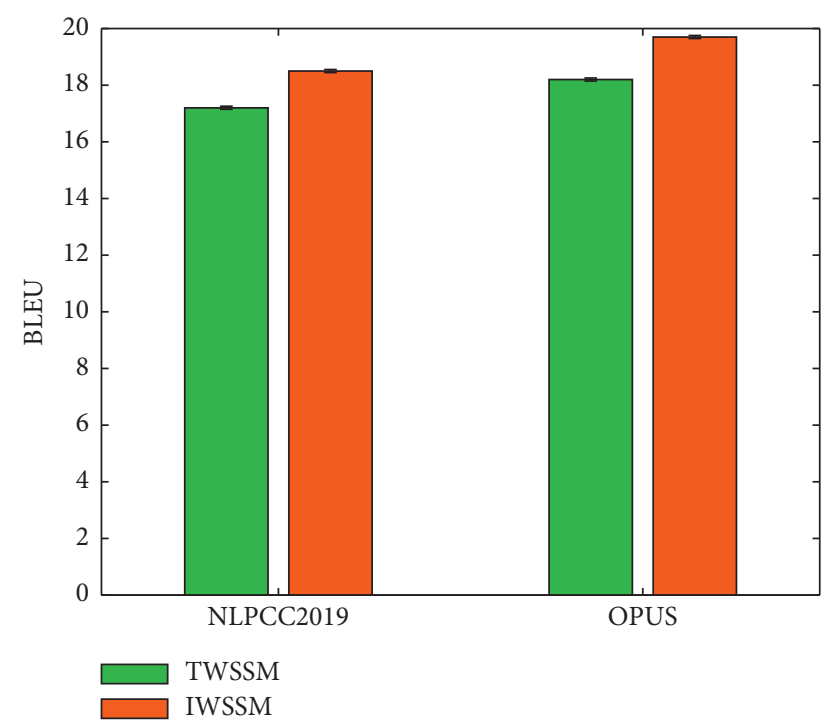

FIGURE 5: Evaluation on improved word sequence segmentation method.

\subsection{Evaluation on Improved Word Sequence Segmentation} Method. As mentioned early, this work proposes an improved word sequence segmentation method. To verify the effectiveness and correctness of this improvement measure, this work will compare and analyze the network performance when the traditional word segmentation method is used and the network performance when the improved word segmentation method is used. The experimental results on NLPCC2019 and OPUS are illustrated in Figure 5. TWSSM is the traditional word sequence segmentation method. IWSSM is the improved word sequence segmentation method.

Obviously, with the improvement of word segmentation methods, the performance of the network can be effectively improved. On NLPCC2019, using the improved word segmentation strategy can get a $1.3 \%$ increase in BLEU. On OPUS, using the improved word segmentation strategy can get a $1.5 \%$ increase in BLEU. This experiment can prove the validity and reliability of improved word sequence segmentation method proposed in this work.

4.6. Evaluation on Improved Attention Structure. As mentioned early, this work proposes an improved attention structure. To verify the effectiveness and correctness of this improvement measure, this work will compare and analyze the network performance when the traditional attention structure is used and the network performance when the

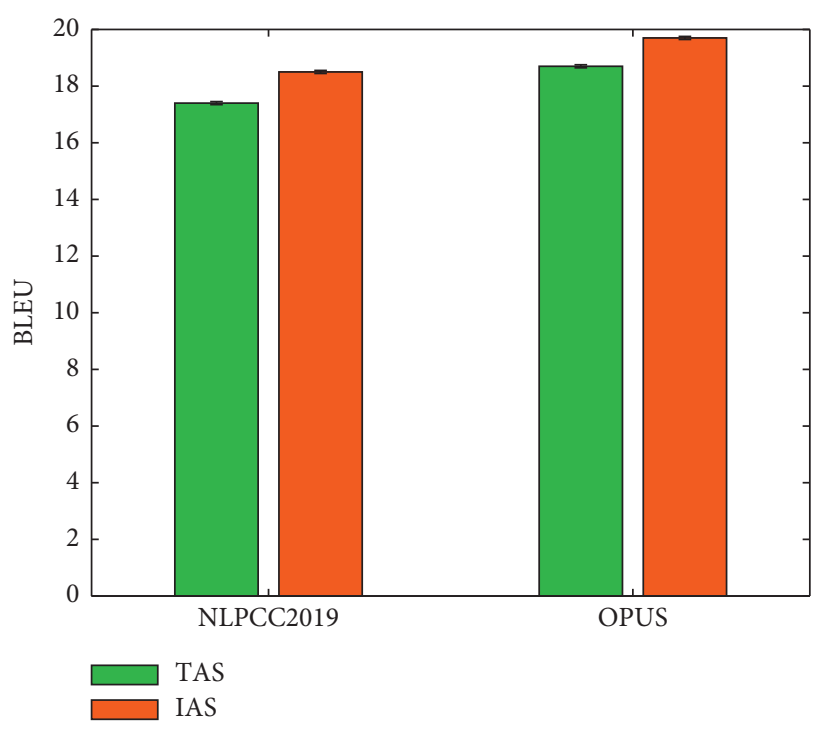

FIgURE 6: Evaluation on improved attention structure.

improved attention structure is used. The experimental results on NLPCC2019 and OPUS are illustrated in Figure 6. TAS is the traditional attention structure. IAS is the improved attention structure.

Obviously, with the improvement of attention structure, the performance of the network can be effectively improved. On NLPCC2019, using the improved attention structure strategy can get a $1.1 \%$ increase in BLEU. On OPUS, using the improved attention structure strategy can get a $1 \%$ increase in BLEU. This experiment can prove the validity and reliability of improved attention structure proposed in this work.

\section{Conclusion}

Since the introduction of deep learning-based neural machine translation, it has replaced traditional phrase-based statistical machine translation method and has become a research hotspot in English translation language processing. As GNMT has surpassed traditional statistical machine translation methods on multiple datasets several times, it has greatly encouraged researchers to study neural machine translation. Based on open-source GNMT, this research studied the application of neural machine translation in English intelligent recognition translation. In view of the current research status of neural machine translation, this work proposed two research contents in different directions. The first is to solve OOV problem of neural machine translation on unregistered words and rare words; this study combined common stemming techniques in English text preprocessing with BPE and proposed a different and improved word sequence segmentation method. Using this method, English text can be segmented into word sequences composed of subword units, and Chinese text can be segmented into character sequences composed of Chinese characters. Secondly, for the prevention of the decoder from experiencing incomplete translation, we adopted an improved attention mechanism that can improve the decoder's 
ability to obtain contextual information. In the improved attention mechanism, a two-layered calculation structure was used to focus on the connection between the context vectors at different moments of the decoder and to improve the ability of attention mechanism to obtain the global context information of the encoder.

\section{Data Availability}

The datasets used during the current study are available from the corresponding author upon reasonable request.

\section{Conflicts of Interest}

The author declares that he has no conflicts of interest.

\section{Acknowledgments}

This work was supported by the Research Project of Social Science Planning in Shandong Province, a study on the translation strategies of the International Image and publicity of the major cities in Belt and Road Shandong Province, 20CWZJ25.

\section{References}

[1] A Fan, S Bhosale, and H Schwenk, "Beyond English-centric multilingual machine translation," Journal of Machine Learning Research, vol. 22, no. 107, pp. 1-48, 2021.

[2] Y. Liu, J. Gu, N. Goyal et al., "Multilingual denoising pretraining for neural machine translation," Transactions of the Association for Computational Linguistics, vol. 8, pp. 726-742, 2020.

[3] M Popel, M Tomkova, and J Tomek, “Transforming machine translation: a deep learning system reaches news translation quality comparable to human professionals," Nature Communications, vol. 11, no. 1, pp. 1-15, 2020.

[4] M Ghazvininejad, V Karpukhin, and L Zettlemoyer, "Aligned cross entropy for non-autoregressive machine translation," in Proceedings of the International Conference on Machine Learning, pp. 3515-3523, Atlanta, GA, USA, July 2020.

[5] L. N Vieira, M O'Hagan, and C O'Sullivan, “Understanding the societal impacts of machine translation: a critical review of the literature on medical and legal use cases," Information, Communication \& Society, vol. 24, no. 11, pp. 1515-1532, 2021.

[6] S. N Khan and I Usman, "A model for English to Urdu and Hindi machine translation system using translation rules and artificial neural network," The International Arab Journal of Information Technology, vol. 16, no. 1, pp. 125-131, 2019.

[7] D. Deng and N. Xue, "Translation divergences in ChineseEnglish machine translation: an empirical investigation," Computational Linguistics, vol. 43, no. 3, pp. 521-565, 2017.

[8] J. Srivastava, S. Sanyal, and A. K. Srivastava, "Extraction of reordering rules for statistical machine translation," Journal of Intelligent and Fuzzy Systems, vol. 36, no. 5, pp. 4809-4819, 2019.

[9] J. Pei, K. Zhong, and J. Li, "ECNN: evaluating a cluster-neural network model for city innovation capability," Neural Computing \& Applications, pp. 1-13, 2021.

[10] C. D. Mellinger, "Translators and machine translation: knowledge and skills gaps in translator pedagogy," The
Interpreter and Translator Trainer, vol. 11, no. 4, pp. 280-293, 2017.

[11] T. Yang, S. Zhao, H. Chen, and B. Chen, "Machine translation system based on deep learning," Journal of Physics: Conference Series, vol. 2030, no. 1, Article ID 012098, 2021.

[12] H. Li and W. Xiong, "Analysis of the drawbacks of EnglishChinese intelligent machine translation based on deep learning," The 2021 International Conference on Machine Learning and Big Data Analytics for IoT Security and Privacy, pp. 104-111, 2021.

[13] Y Tian, S Khanna, and A Pljonkin, "Research on machine translation of deep neural network learning model based on ontology," Informatica, vol. 45, no. 5, 2021.

[14] K. L. Kermanidis, "NoDeeLe: a novel deep learning schema for evaluating neural machine translation systems," TRITON, vol. 37, 2021.

[15] C. Park, Y. Yang, K. Park, and H. Lim, "Decoding strategies for improving low-resource machine translation," Electronics, vol. 9, no. 10, p. 1562, 2020.

[16] N Yang, S Liu, and M Li, "Word alignment modeling with context dependent deep neural network," in Proceedings of the 51st Annual Meeting of the Association for Computational Linguistics, pp. 166-175, Sofia, Bulgaria, August 2013.

[17] L Cui, D Zhang, and S Liu, "Learning topic representation for SMT with neural networks," in Proceedings of the 52nd Annual Meeting of the Association for Computational Linguistics, pp. 133-143, Maryland, MA, USA, June 2014.

[18] P Li, Y Liu, and M Sun, "A neural reordering model for phrase-based translation," in Proceedings of the COLING 2014, The 25th International Conference on Computational Linguistics: Technical Papers, pp. 1897-1907, Dublin, Ireland, August 2014

[19] Y Bengio, R Ducharme, and P Vincent, "A neural probabilistic language model," Journal of Machine Learning Research, vol. 3, no. 2, pp. 1137-1155, 2003.

[20] N Kalchbrenner and P Blunsom, "Recurrent continuous translation models," in Proceedings of the 2013 Conference on Empirical Methods in Natural Language Processing, pp. 1700-1709, Washington, DC, USA, October 2013.

[21] K Cho, B van Merriënboer, and C Gulcehre, "Learning phrase representations using RNN encoder-decoder for statistical machine translation," in Proceedings of the 2014 Conference on Empirical Methods in Natural Language Processing, pp. 1724-1734, Doha, Qatar, October 2014.

[22] I Sutskever, O Vinyals, and Q. V Le, "Sequence to sequence learning with neural networks," Advances in Neural Information Processing Systems, pp. 3104-3112, 2014.

[23] D Bahdanau, K Cho, and Y Bengio, "Neural machine translation by jointly learning to align and translate," 2014, https://arxiv.org/abs/1409.0473.

[24] M. T Luong, H Pham, and C. D Manning, "Effective approaches to attention-based neural machine translation," in Proceedings of the 2015 Conference on Empirical Methods in Natural Language Processing, pp. 1412-1421, Lisbon, Portugal, September 2015.

[25] Y Wu, M Schuster, and Z Chen, “Google's neural machine translation system: bridging the gap between human and machine translation," 2016, https://arxiv.org/abs/1609.08144.

[26] J. Gehring, M. Auli, and D. Grangier, "Convolutional sequence to sequence learning," in Proceedings of the 34th International Conference on Machine Learning, pp. 1243-1252, Sydney, Australia, August 2017. 
[27] A. Vaswani, N. Shazeer, and N. Parmar, "Attention is all you need," Advances in Neural Information Processing Systems, pp. 5998-6008, 2017.

[28] G Lample, M Ott, and A Conneau, "Phrase-based \& neural unsupervised machine translation," in Proceedings of the 2018 Conference on Empirical Methods in Natural Language Processing, pp. 5039-5049, Brussels, Belgium, November 2018.

[29] C Gulcehre, S Ahn, and R Nallapati, "Pointing the unknown words," 2016, https://arxiv.org/abs/1603.08148.

[30] J Chung, K Cho, and Y Bengio, "A character-level decoder without explicit segmentation for neural machine translation," 2016, https://arxiv.org/abs/1603.06147.

[31] M. T Luong and C. D Manning, "Achieving open vocabulary neural machine translation with hybrid word-character models," 2016, https://arxiv.org/abs/1604.00788.

[32] R Sennrich, B Haddow, and A Birch, "Neural machine translation of rare words with subword units," 2015, https:// arxiv.org/abs/1508.07909.

[33] P Gage, "A new algorithm for data compression," Users Journal, vol. 12, no. 2, pp. 23-38, 1994. 